\title{
Dark Matter and Quantum Entanglement Decoded
}

\author{
Bhushan Poojary
}

\begin{abstract}
Quantum entanglement is a physical phenomenon that occurs when pairs (or groups) of particles are generated or interact in ways such that the quantum state of each member must subsequently be described relative to the other, Repeated experiments have verified that this works even when the measurements are performed more quickly than light could travel between the sites of measurement. In this paper, how the communication between entanglements particles occurs, is explained with help of quantization of space and holographic principle of our universe. Dark matter and dark energy too is explained with the help of quantization of space and holographic principle, that's why it is hard to detect as of now because it is an effect from holographic plate on our universe.
\end{abstract}

Index Terms-Holographic principle, quantization of space, quantum entanglement, dark matter, dark Energy.

\section{QuANTIZATION OF SPACE}

In paper Fine Structure Decoded [1], vacuum is divided into equally spaced, real and imaginary space and it covers whole region. This means there is no void space between them. A cell here is represented by rhombic dodecahedron as shown in Fig. 1. Rhombic dodecahedron was chosen because it is tessellating in nature, it is very close to shape of sphere and covers maximum volume.

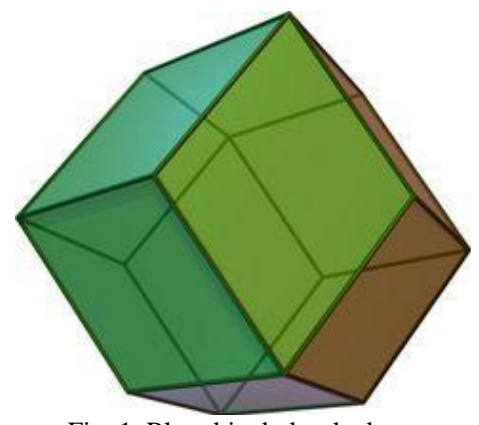

Fig. 1. Rhombic dodecahedron.

Structure of space is shown below in Fig. 2. Space is made of alternating real and imaginary space.

\section{Structure OF Electron}

Electron in fine structure decoded paper is described as imaginary space confined inside real space.

In Fig. 3 you can see blue space (imaginary space) confined inside green rhombic dodecahedron (real space). Yellow spot in between is called zero space formed by combination of real and imaginary space, which moves around.

Manuscript received February 12, 2014; revised April 24, 2014.

Bhushan Poojary is with NIMS University, India (e-mail: bhushanpoojary@gmail.com).

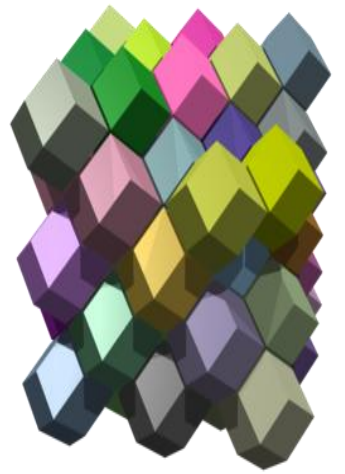

Fig. 2. Rhombic dodecahedron structure occupying complete space.

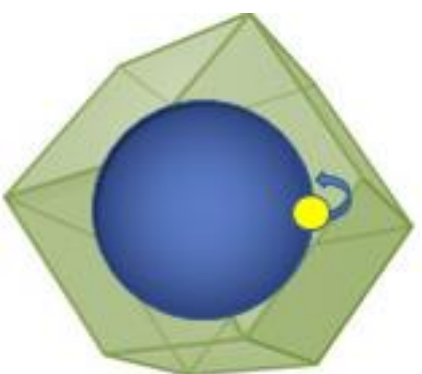

Fig. 3. Structure of electron.

\section{STRUCTURE OF POSITRON}

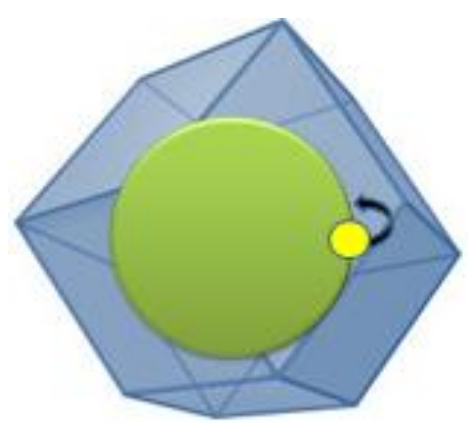

Fig. 4. Structure of positron.

Positron in fine structure decoded paper is described as real space confined inside imaginary space.

In Fig. 4 you can see green space (real space) confined inside blue rhombic dodecahedron (real space). Yellow spot in between is called zero space formed by combination of real and imaginary space, which moves around.

\section{HOLOGRAPHIC PRINCIPLE OF UNIVERSE AND QuANTIZATION OF SPACE}

The holographic principle is a property of quantum gravity and string theories that states that the description of a volume of space can be thought of as encoded on a boundary to the region [2] — preferably a light-like boundary like a 
gravitational horizon. First proposed by Gerard't Hooft, it was given a precise string-theory interpretation by Leonard Susskind who combined his ideas with previous ones of Hooft and Charles Thorn. As pointed out by Raphael Bousso, Thorn observed that string theory admits a lower-dimensional description in which gravity emerges from it in what would now be called a holographic way.

In a larger sense, the theory suggests that the entire universe can be seen as a two-dimensional information structure "painted" on the cosmological horizon, such that the three dimensions we observe are only an effective description at macroscopic scales and at low energies. Cosmological holography has not been made mathematically precise, partly because the cosmological horizon has a finite area and grows with time.

Now if we considered quantization of space in terms alternating placed rhombic dodecahedron, we can correlate it with holographic projection of 2D surface on our universe. This 2D surface also should be quantized too, may or may not correspond to hexagonal area or areas in 2D surface in holographic surface.

\section{PROCESS OF ENTANGLEMENT}

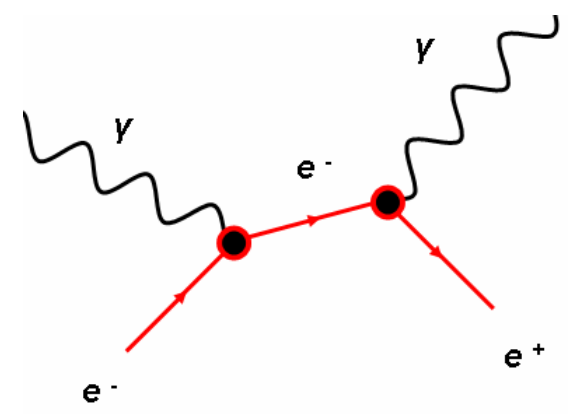

Fig. 5. Entangled electron and positron generation from 2 photons.

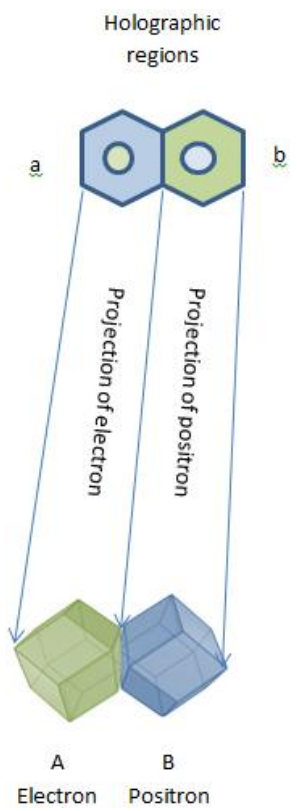

Fig. 6. Projection of holographic boundary while entanglement particles are created.

Entanglement is usually created by direct interactions between subatomic particles. These interactions can take numerous forms. One of the most commonly used methods is spontaneous parametric down-conversion to generate a pair of electrons and positron. For example if we take example of two photon colliding scenario which generates pair of electron and positron as shown in Fig. 5.

From Fig. 5 it is clear that entangled electron and positron pair is created simultaneously and very close to each other.

If we consider this in quantization of space, electron and positron will be created in adjacent cell in real and imaginary space, this event is actually recorded in adjacent cells in 2D hologram surface on time of creation.

\section{How COMMUNiCATION HAPPENS BETWEEN ENTANGLED PARTICLES?}

As explained above entangled particles are generated in adjacent region (one in real space and one in imaginary space), this event in our universe is a holographic view of $2 \mathrm{D}$ surface, this $2 \mathrm{D}$ surface too is quantized like it is quantized in our universe, which means this 2 regions where the entangled particles were created in our universe will also be associated to $2 \mathrm{D}$ definite region in $2 \mathrm{D}$ surface in holographic horizon. We will call this region as origin of entanglement.

When these entangled particles are created they start moving in different direction. When any property of one entangled particle is altered same time in holographic region (origin associated when entangled particles were created) associated with creation of these particles also alters accordingly. As the first holographic region is very closely spaced to paired holographic region at the holographic origin, paired holographic region too alters in accordingly. This alteration modifies the property of paired particle because it is holographic image of this $2 \mathrm{D}$ surface.

Thus it is able to communicate between each other instantaneously even when they are not directly connected.

\section{DARK MATTER REVELED}

Dark matter is a type of matter hypothesized in astronomy and cosmology to account for a large part of the mass that appears to be missing from the universe. Dark matter cannot be seen directly with telescopes; evidently it neither emits nor absorbs light or other electromagnetic radiation at any significant level. It is otherwise hypothesized to simply be matter that is not reactant to light [3].

According to the Planck mission team, and based on the standard model of cosmology, the total mass-energy of the known universe contains $4.9 \%$ ordinary matter, $26.8 \%$ dark matter [4].

But still what is dark matter made up of is still not explained, even though it is more than $25 \%$ of observable universe.

Now consider case of entanglement where 2 entangled particles are created and registered 2 regions in holographic plane. If we compare these 2 regions with normal regions in holographic plane, registered regions are different from other holographic plate regions which are empty.

In our universe matter curves space, similarly these registered 2 regions too should curve the holographic space surrounding it as show below in Fig. 7. 


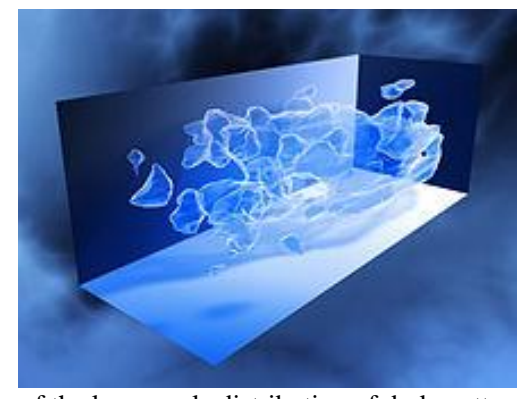

Fig. 6. D map of the large-scale distribution of dark matter, reconstructed from measurements of weak gravitational lensing with the Hubble Space Telescope [5].

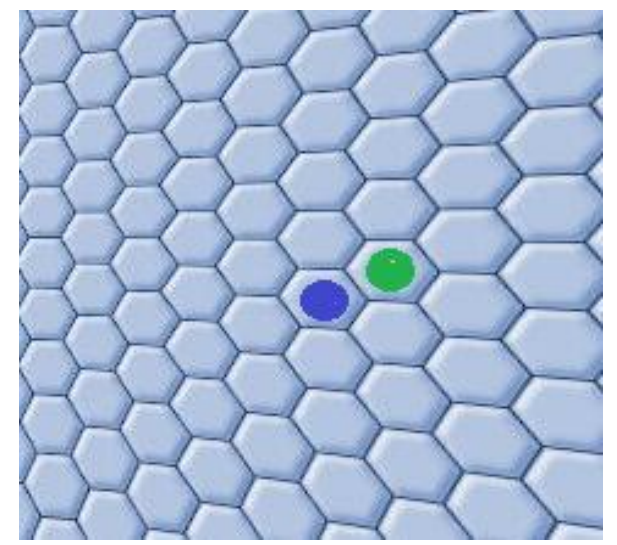

Fig. 7. Shows two regions registered in holographic plane when 2 entangled particles are created, this curves the holographic regions surrounding them.
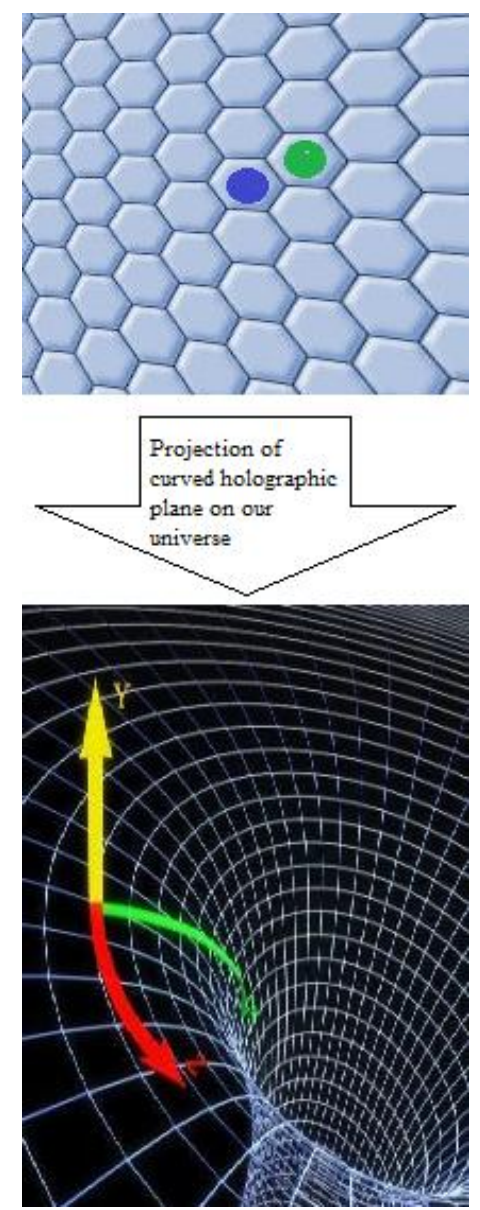

Fig. 8. Projection of curved holographic plane in our universe leads to curvature of space and time.

As we know whatever happens in holographic region is replicated in our universe too (shown in Fig. 8). So this curved holographic surface, projects curvature in space time in our universe as well. This curvature is same as generated due to mass on our universe.

That's why dark matter is difficult to find because it real existence is not in our universe but in holographic planes.

Abundance of dark matter can be explained with help of big bang.

In big bang lot of matters were created (and some anti-matter) [4], this creation also registered regions in holographic planes and thus dark matter came into existence. So what we see now is remnant of big bang in holographic plane which creates curvature of space time which leads to gravitation in our universe [5].

To generate dark matter one needs to create entangled pair and quickly send photons where these 2 pair were created and we can observe if the space was curved by detecting red shift or refraction of photon when passing through his region. We should send detectable photons very quickly because our whole galaxy is moving at high speed compared to quantized space. This experiment will prove that dark matter can be generated in laboratory and can be detected too, and will prove its correlation with entangle particles.

General theory of relativity formula needs to address this curvature of space which comes due to dark matter even though actual matter is not physically present to get desired results. This was taken into consideration in general theory of relativity as cosmological constant but later discarded as biggest mistake of Einstein. But if we consider that it terms of registered spaces in our universe we can account to dark matter in general theory of relativity.

\section{DARK ENERGY REVELED}

As discussed above we know that when matter moves through the registered space in our universe it goes through space time curvature and it the motion of the matter is affected due to it, in this we should also note that registered space too gets affected by incoming matter, this indirectly affects the motions of the matter antimatter associated with this registered region.

So from above we can say that dark energy and dark matter both come from registered spaces in our universe.

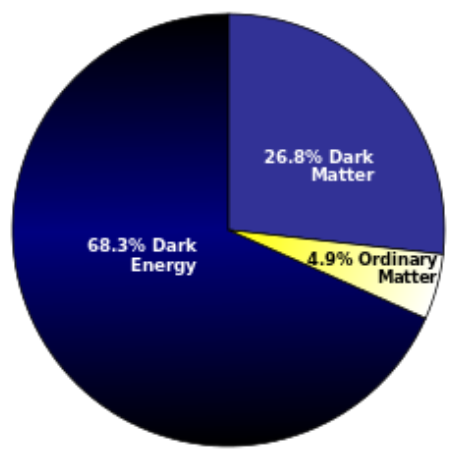

Fig. 9. Distribution of dark energy and dark matter in our universe.

From Fig. 9 we can see that dark energy makes around $68.3 \%$ [6] of current universe, which is a big factor.

Dark energy is thought to be very homogeneous, not very dense and is not known to interact through any of the fundamental forces other than gravity [7], this means when big bang occurred the registered spaces were arranged 
homogenously. This registered spaces has a binding energy as predicted by Einstein, volume of space has an intrinsic energy associated with it. Einstein had predicted that "cost of having space": that is, a volume of space has some intrinsic, fundamental energy [8]. This finding is close to what Einstein had predicted but it is effect of holographic principle registered spaces in our universe.

From the statistics collected we know our universe is expanding in accelerated growth due to dark energy [9], expansion of universe vs time is shown below in Fig. 10 [10].

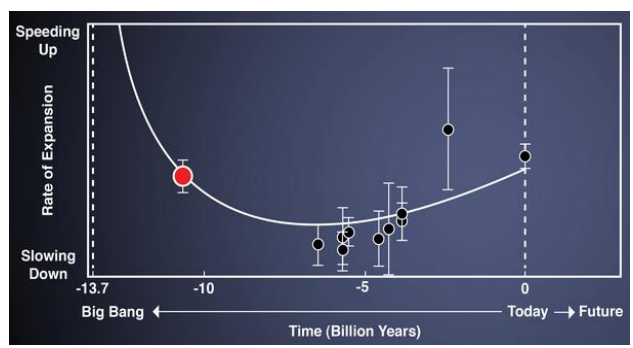

Fig. 10. Expansion curve of our universe.

From above chart is clear that universe was slowing down initially and later it picked up speed due to dark energy. Dark energy and Dark matter go hand in hand. Both do not exists directly in our universe but it effect is observed, more the matter, anti-matter and photons pass through dark matter, more the potential energy is transferred to registered pair associated with those registered region. As universe spread gravitational forces due to individual galaxy goes down and matter goes through dark matter, and thus gains energy (similar to ball left from top to reach earth, where potential energy is converted into kinetic energy) this keeps the universe expanding.

Lot of dark energy and dark matter in our universe implies that in early universe most of the matter and anti-matter got annihilated, which now acts as a registered region, which creates an effect like dark matter and dark energy.

\section{CONCLUSION}

Holographic principle and quantization of space is real cause of entanglement, communication between entangled particles which takes place through holographic principle and entangled particles/photons, which are not directly connected as predicted by worm-hole theory. Dark matter is projection of curved holographic plane in our universe. Dark energy is due potential energy difference between matter/photon and registered pair region in our universe which are projections of holographic plates.

\section{REFERENCES}

[1] B. Poojary, "Fine Structure Decoded," International Journal of Applied Physics and Mathematics, vol. 2, no. 4, pp. 244-249, 2012.

[2] S. Leonard, "The World as a Hologram," Journal of Mathematical Physics, vol. 36, no. 11, pp. 6377-6396, 1995.

[3] V. Trimble, "Existence and nature of dark matter in the universe," Annual Review of Astronomy and Astrophysics, vol. 25, pp. 425-472, 1987.

[4] Kolb and Turner, chapter 6, 1988,

[5] Nobel Prize Biography, Nobel Prize Biography, Nobel Prize.

[6] E. Saul, 7 The Coded Universe: The Path to Eternity.

[7] S. K. Basu, 8 Encyclopaedic Dictionary of Astrophysics.

[8] D. W. Lipp, 6 I have become Space.

[9] L. Amendola and T. Shinji, Dark Energy: Theory and Observations.

[10] Z. Rostomian, Lawrence Berkeley National Laboratory, and Nic Ross, BOSS Lyman-alpha team, Berkeley Lab.

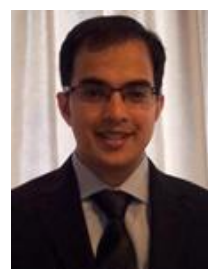

Bhushan Poojary is pursuing bachelor's degree from NIMS university. He was born in Mumbai on 26 July 1984. He has done bachelor of engineering in electronics and telecom from Mumbai University in year 2006.

$\mathrm{He}$ is currently working as a software engineer in Credit Suisse in Mumbai.He has published paper Fine structure decoded in IJAPM vol. 2, no. 4, 2012. He has more than 8 years of experience in Information 